\title{
BLOCKCHAIN TECHNOLOGY: ENERGY EFFICIENCY AND ETHICAL COMPLIANCE
}

\author{
Yudi Fernando ${ }^{1, *}$ and Rubakanthan Saravannan² \\ ${ }^{1}$ Faculty of Industrial Management, Universiti Malaysia Pahang, 26300 Pahang, Malaysia. \\ ${ }^{2}$ Tenaga Nasional Berhad, 25000 Pahang, Malaysia.
}

\begin{abstract}
Blockchain technology can disrupt almost every industry due to its ability to create a decentralized tamper-proof ledger network and carry out transactions without the need of a trusted third-party intermediary. One of the significant drawbacks of blockchain technology is the energy consumption surrounding its application. The problem has been discussed extensively, with proposals focusing on strengthening its consensus protocol and integrating renewable energy sources to minimize its carbon footprint. To boost blockchain's adaptability in all sectors, the technology itself needs to look for less energy-intensive alternatives to boost its ethical and industrial compliance. As the energy sector is currently undergoing a revamp from its age-long oneway power network towards a decentralized grid system with distributed generation and storage. The adoption of blockchain technology must work hand in hand with the energy industry to make itself energy efficient and competitive in the long term.
\end{abstract}

ABSTRACT - Blockchain technology can disrupt almost every industry due to its ability to create
a decentralized tamper-proof ledger network and carry out transactions without the need of a
trusted third-party intermediary. One of the significant drawbacks of blockchain technology is the
energy consumption surrounding its application. The problem has been discussed extensively, with
proposals focusing on strengthening its consensus protocol and integrating renewable energy
sources to minimize its carbon footprint. To boost blockchain's adaptability in all sectors, the
technology itself needs to look for less energy-intensive alternatives to boost its ethical and
industrial compliance. As the energy sector is currently undergoing a revamp from its age-long one-
way power network towards a decentralized grid system with distributed generation and storage.
The adoption of blockchain technology must work hand in hand with the energy industry to make
itself energy efficient and competitive in the long term.

ARTICLE HISTORY

Received: 11-1-2021

Revised: 18-2-2021

Accepted: 9-3-2021

\section{KEYWORDS}

Energy efficiency, Blockchain technology, Renewable energy, Ethical compliance, Energy-Intensive

\section{INTRODUCTION}

Adaptation of new technology such as blockchain raises many challenges that need to be ironed out before accepted by the commercial and industrial sectors. This paper has discussed energy consumption, blockchain technology adoption, ethical compliance, and areas that could improve its energy efficiency. Blockchain provides a secure environment where transaction content cannot be changed, and it is possible for nodes to anonymously engage in the application. It includes a distributed ledger, peer-to-peer connectivity, cryptography, and other reputable technologies. Thus, blockchain technology can be widely adopted in many fields, such as medical systems, financial, food, transportation, and the supply chain (Zhang \& Lee, 2020). Technological capability is critical for the success of manufacturing firms (Fernando et al., 2020a).

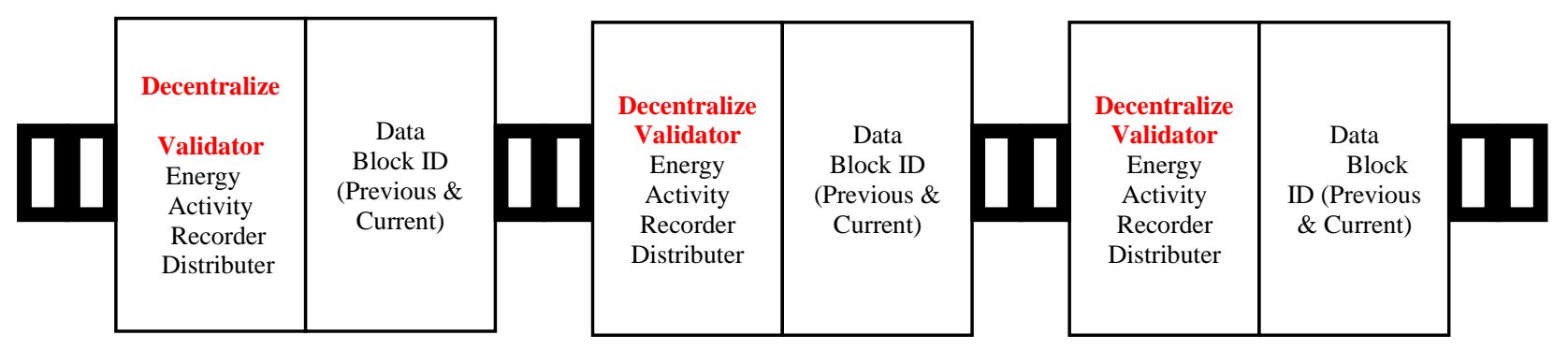

Figure 1: Basic build of a blockchain

Figure 1 shows the basic build of a blockchain system for energy efficiency. As the name implies, blockchain is a chain of blocks stringed together, with each block consisting of data on its previous and current identification. The block is an essential component of the technology as it records the energy activity/ consumption, distributes and authenticates transactions. As a combination of numbers/letters interconnects the chain, it is tamper-proof, and any changes to one of the blocks will render the entire chain void. Each transaction is encrypted and distributed to several (peer-to-peer) computers, which store the data locally. The users are not able to manipulate the data entry. It is the main element of ethical report compliance.

The data stored in a block is checked using algorithms that add a unique hash to each block that serves as its identity. This hashtag is a series of numbers and letters generated based on information stored in the data block. If any transaction information is subsequently altered due to manipulation or transmission errors, the algorithm running on the altered block will no longer generate the correct hash and, therefore, record an error (Khatoon et al., 2019). This continuous verification process, also referred to as 'mining', is carried out by blockchain users paid for their services based on the computing power they contribute.

The verification process ensures that all participants can contribute to the extension of the blockchain. Once a block is checked and implemented, there can be no subsequent changes to the block in question. It is one justification that 
blockchain can legally record direct peer-to-peer transactions between individuals or organizations without intermediary services (PricewaterhouseCoopers 2016). A blockchain agreement is that all nodes hold the exact copy of the distributed ledger, making it immutable. The rising demand for integrity practices in their supply chains can improve accurate carbon disclosure, report carbon verification, and others (Mohter \& Fernando, 2020).

This paper aims to conceptualize blockchain technology adoption to achieve energy efficiency with ethical compliance. Blockchain adoption has been covered in a different type of industrial and countries settings such as healthcare (Balasubramanian et al., 2021), online community (Zheng \& Boh, 2021), operation and manufacturing (Lohmer \& Lasch, 2020), food distribution (Bumblauskas et al., 2020), and halal supply chain (Fernando et al., 2020b,c). This paper has its uniqueness as the previous studies rarely discuss how blockchain technology adoption can assist in the energy report's ethical compliance. It is time to craft this paper as Malaysian manufacturing firms face pressure to report the carbon footprint (Fernando et al., 2021). In the Malaysian setting, Naina et al. (2019) and Shaharudin et al. (2020) argued that more initiatives are needed to strengthen environmental supply chain integrity reports. Besides that, the key personnel in manufacturing firms are expected to understand supply chain integration and supply chain responsiveness to handle operations issues (Fernando \& Wulansari, 2020).

\section{LITERATURE REVIEW}

Every node can act as a host/server in this network and exchange data with other nodes to achieve agreement. In some instances, there may be malicious nodes, which could affect or destroy its chain integrity. An outstanding protocol should reduce the frequency of such events or avoid any impact on the result (Zhang \& Lee, 2020). The protocol that the network has implemented must be appropriate for its blockchain system type. Blockchain is divided into three categories (Wu \& Tran 2018) which are:

i. Public blockchain: Enables any person to access information, provide sufficient confirmation of transactions and engage in consensus procedures. It implies that the nodes can be anonymous, producing a perfect decentralized network. Bitcoin and Ethereum are the most well-known public blockchain in the present application.

ii. Consortium blockchain: Implements pre-set nodes to manage its consensus procedures. The blockchain may restrict access for the public, particular participants, or follow a hybrid access method, making the network "partial decentralized". Blockchain networks like Alliance and Hyperledger are examples of consortium blockchain.

iii. Private blockchain: Operates so that one person controls the write access process; hence, access authorization is limited. This type of platform is widely used for the management and auditing of individual company's databases. In order to achieve consensus on a transaction, a trustworthy node is required. It will offer a safer option for firms preparing to adopt blockchain since it is managed by a trusted entity and is commonly shared with the authenticated database. Coin Science and Eris Industries are businesses that specialize in delivering enterprise blockchain services.

\section{Blockchain Energy Consumption Drawback}

As interest in blockchain-related technologies increases, there has been an upsurge in energy demand to support the technology. In the last five years, global power demand forecasts for blockchain-based technologies have ascended, from 2500 to 7670 megawatts (Clark \& Greenley, 2019). Future development of the technology will significantly impact energy demand and resulting in increased emissions of carbon dioxide. Blockchain and cryptocurrency experts at PwC have reported that the overall power demand for blockchain-based applications servers is 22 terawatt-hours (TWh) of energy usage annually (PwC, 2016). That is almost similar to the overall electricity consumption of a country such as Ireland. For instance, Google has a consumption of about 5.7 TWh of energy to power its global operations (Baraniuk, 2019). Another worrying problem is that miners raise their energy intake by five folds yearly, which has triggered a huge spike and shows no slowdown.

\section{Areas of Improvement}

As energy consumption issues continue to build a more cynical view worldwide, society has demanded more on blockchain technology. It is essential to look into making the technology attractive and ease its adoption commercially. Adequate consideration should be given to some of the areas below to make blockchain energy-efficient and sustainable (Truby, 2018).

1. Implement less energy-intense protocol to reach consensus.

2. Incorporate renewable energy sources to increase efficiency and reduce carbon emissions, thus creating a sustainable technology.

3. Develop regulations and incentives to curb the use of inefficient hardware and energy resources. 


\section{Energy-efficient Algorithms}

A significant process in blockchain technology is determining which node generates the next block in the chain. This process is conducted using a validation process that reaches a consensus on the distributed ledger's content. Consensusbased validation is achieved directly via a peer-to-peer network between individual parties. Useful nodes are financially compensated, which motivates them to continue validating and adding blocks to the network.

Four key factors lead to blockchain energy consumption: Hardware processing capacity, network hash rate, complexity, and hardware thermal regulation. Implementing an energy-efficient mechanism is significant, as the consensus algorithm directly affects all four of these variables (Clark, \& Greenley, 2019). Many consensus protocols can be implemented in the blockchain network and have their own set of characteristics. The most common ones are proof of work, proof of stake, and proof of authority.

\section{Proof of Work (PoW)}

The most widely used consensus mechanism in blockchain technology is PoW, which is based on a validator sometimes referred to as a miner. These miners are tasked with solving difficult cryptographic problems to have the right to expand another block in the chain. The miner is then paid in the form of a financial reward for the completed task. Ideally, this provides an ideal decentralized peer-to-peer network that enables anonymous authentication of transactions. The complexity and hash rate will increase accordingly as blockchain networks expand. The results in a rivalry between validators require high power computing resources to solve the increasingly complex algorithm (Monrat et al., 2019). The benefit of PoW is that it allows anybody with hardware to access the network. It is partly a miner and protects the credibility of the blockchain.

Although this choice of consensus protocol is widely utilized, its need for high computational resources and possible attacks by organizations with $51 \%$ hardware power constitutes a significant concern (Yaga et al., 2018). Blockchains such as Ethereum, Monero, and Bitcoin are examples of large permissionless or public platforms. When a network is extensive and decentralized, the PoW algorithm will be increased in difficulty, contributing to higher energy consumption and transaction slows down (Eurelectric Report, 2018). PoW's main critique is that massive amounts of natural resources are wasted, and hard to reach a consensus of solution (Andoni et al., 2019). For instance, the computing power needed to maintain blockchain technology such as Bitcoin consumes the same amount of energy as Switzerland's entire country. According to Cambridge Electricity Consumption Index (CBECI), Bitcoin cryptocurrency's annual consumption is around $60.45 \mathrm{TWh}$, which renders it the 41st most energy-consuming country if it ranks among other nations (Cuthbertson, 2019). These are massive figures of energy consumption for a technology in its growth stage and it is not sustainable in the near future.

\section{Proof of Stake (POS)}

The energy drain of the PoW mechanism, which is well documented, has steered towards proposals of other substitutes such as the Proof of Stake. The algorithm replaces hardware-related tasks with an arbitrary selection procedure, where the probability of selection is proportionally linked to a validator stake in the network. In other words, the likelihood of a user being allowed to create a block depends on how much has been put at stake by the user. This approach could lead to speedier blockchains transactions with less intense energy requirements and decrease the chances of an attack by nodes with majority hardware strength (Andoni, et al. 2019).

With this model, there is no need to carry out resource-intensive computations requiring hardware, electricity, and time that was a prerequisite in the PoW protocol as this consensus model requires fewer resources than the traditional ones. Some blockchain networks may opt to forego the traditional block creation rewards towards a standardized fee system. The method blockchain networks implement validators' selection may vary with coin maturing, delegated systems, or round-based vote casting mechanisms. Whatever the strategy implemented, new blocks are more likely to be released by users with more stake in a network (Monrat et al., 2019).

This consensus protocol's key benefit is that it needs much less computational resources than PoW but may potentially entail attacks by those with ample financial power (Yaga et al., 2018). Ethereum, one of the leading blockchain platforms for developers and firms, is contemplating moving from its present PoW protocol to the PoS algorithm. The move is predicted to cut the energy consumed per Ethereum transaction by as much as 99 percent (IEEE, Jan 2019).

\section{Proof of Authority (POA)}

For authorized consortium and private blockchain platforms with a high level of trust, PoA could be explored. In this consensus model, it is possible to have approved nodes in a platform operating as the elected authority, and these validators run software that allows transactions to be placed on the distributed ledger. Simultaneously, the protocol is computerized and does not require continuous monitoring by the elected authority. The PoA blockchain's integrity requires validators' hardware to be uncompromised and safe from any malicious acts. Authority nodes may lose credibility by behaving so that the blockchain network users disagree with, just as they can gain credibility by acting with integrity. The lower the credibility, the less likely a block would be published by the node (Monrat et al., 2019).

As the PoA approach consists of elected nodes, the protocol is more centralized and prone to attack than other models but has the added benefit of faster transaction speeds. For example, Tobalaba Energy Web Foundation, which operated a PoA protocol network, acts as a validator for adapted blockchain applications. This firm operates as a validator for some 
major energy companies such as Engie, Shell, Statoil, and Tepco. The average time required to validate a transaction by these networks runs in the range of three to four seconds, which is fast by blockchain standards (Eurelectric Report 2018).

\section{Renewable Energy}

With the rise in electricity demand to support blockchain technology, energy efficiency will play a key role in reducing the overall amount of power required to meet these demands. According to the Paris Agreement objectives, efficiency and renewables work hand in hand while playing a key role in decarbonizing the global energy system. In 2015 , the Sustainable Development Goals (SDGs) provided a framework for global firms to accomplish a sustainable future with their energy resources. The goal includes the significantly rising share of renewable sources in the world energy mix and doubling energy efficiency rates (Gielen et al., 2019). As renewable energy provides higher output than traditional energy sources, increasing its energy mix penetration will improve overall energy efficiency.

Traditionally, Malaysia has been dependent on conventional power generation, with oil being the primary energy source in the early 1980s. However, in 1981 the government adopted a four-fuel diversification program incorporating other nonrenewable sources of energy. Such policies seek to reduce oil reliance as the critical source for electricity generation and ensure future energy sustainability by utilizing other nonrenewable energy sources (Sharvini et al., 2018). This policy was followed in 2001 by the Five-Fuel Diversification Policy, with the introduction of renewable energy (RE) as the fifth fuel source, and it signaled the government's intention to use renewable energy (Tan et al., 2013).

The Malaysian government recently announced a revision of the national RE target to a more ambitious 20 percent penetration rate by 2025 . Around 50 percent of the target will come from solar and the rest from non-solar technologies. Solar is seen as the most convenient RE technology to deploy in Malaysia due to the abundance of sun irradiation and its lower capital requirement. The other non-solar technologies require substantial government support and capital to ensure project viability (Abdullah et al., 2019).

Sustainable Energy Development Authority (SEDA) Malaysia has introduced several solar initiatives and programs to encourage Malaysia's renewable uptake (SEDA, 2020). Currently, there are three main solar energy programs offered in Malaysia by SEDA. These programs are the self-consumption (SeLCO), Net Energy Metering (NEM), and Large Scale Solar (LSS) plant scheme. The SeLCO and NEM programs are well suited for blockchain adaptation as it provides a decentralized and sustainable electricity source to meet the technology's daily energy demand. Figure 2 and Figure 3 show the SeLCO and NEM operations based on SEDA (2020) concept.

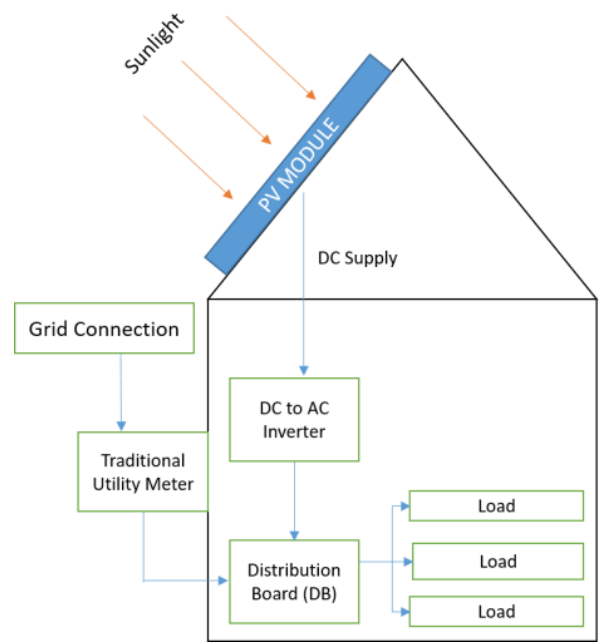

Figure 2: SeLCO operation

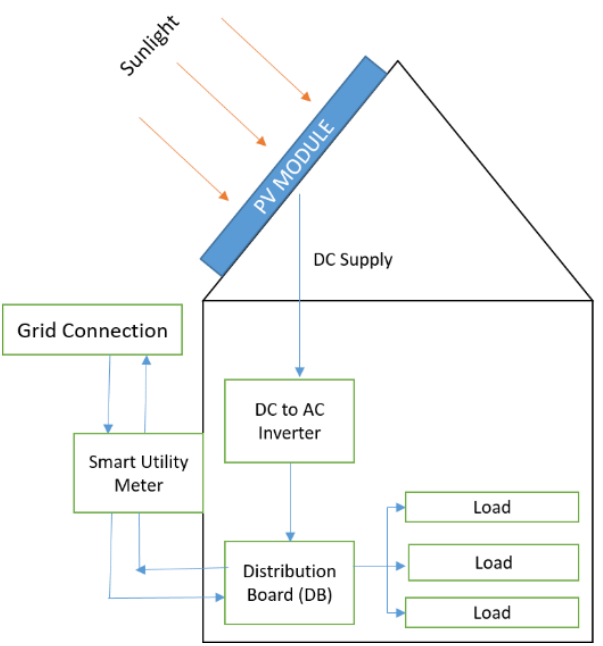

Figure 3: NEM operation

\section{Self-Consumption (SeLCO)}

In this mechanism, consumers must install a solar photovoltaic (P.V.) system for their use. Electricity exports to the grid are not permitted in the event of excess energy generation. It is the consumer and registered electrical contractor's responsibility to ensure no export to the grid of electricity generation.

\section{Net Energy Metering (NEM)}

The concept of NEM is similar to SeLCO, with the critical difference being the bi-directional meter, which replaces the traditional one-way metering system. This meter can track excess energy that is exported out from the prosumers' premises. The energy produced from the solar photovoltaic system must be first be consumed; only then any excess is allowed for export to other prosumers or the energy utility. The NEM system provides consumers with the same tariff for selling and buying electricity and is expected to draw more customers to install a solar rooftop system. 
Besides initiatives by the government, technology has also been favorable for the sector. P.V. module prices have fallen aggressively, and its efficiency is continuously improving. Prices of inverters and interconnection pieces of equipment are also decreasing. It has contributed toward an overall reduction of system cost of constructing a P.V. system, and developers can now offer competitive energy rates. Improvements in technology, economies of scale and manufacturing experience, and supply chain efficiency allow solar panel manufacturers to decrease pricing.

This rate is unparalleled by other competing renewable energy sources, making the technology highly attractive (Irena, 2016). Solar energy can be viewed as one of the main drivers in implementing an efficient, decentralized, and robust energy system in Malaysia. These solar programs and the low capital cost could allow blockchain technology to utilize renewables to cater to its energy demand, reducing the carbon footprint. The SelCO and NEM program has the potential to improve the penetration of RE further. The scheme allows prosumers to exist in the energy system and carry out trading of green energy.

Further incorporation of battery cells at the prosumer side could cater for the intermittent low sun irradiation periods, which could reduce reliance on conventional backup energy from the grid. Cryptosolartech, Spain's largest Bitcoin miner, has already led the charge towards renewable sources. The firm is constructing a $300 \mathrm{MW}$ solar plant, which will make its future blockchain-based operations sustainable and reduce environmental impacts (CCN, 2020). Figure 4 shows the mechanism of blockchain regulation and it will be discussed in the next section.

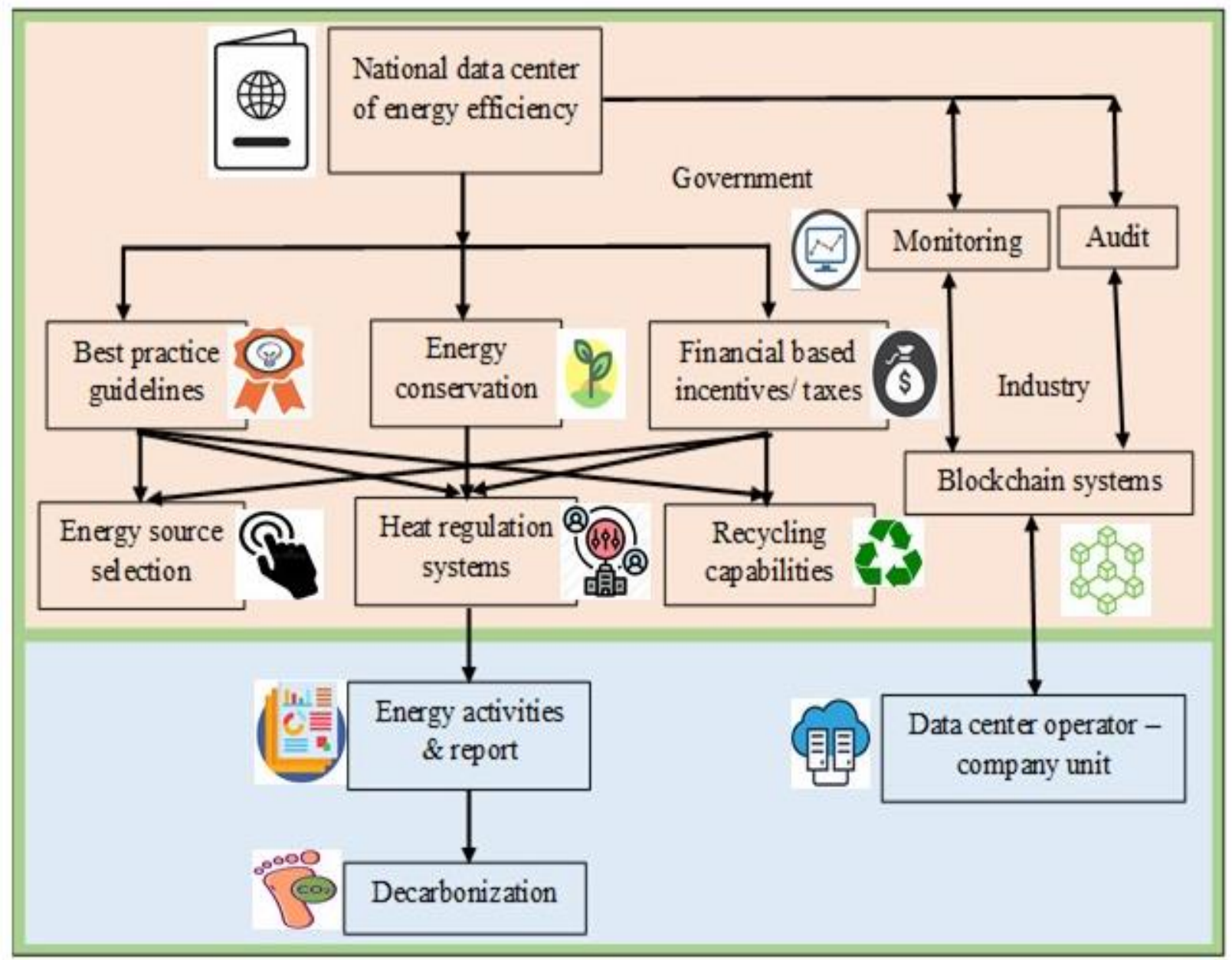

Figure 4: The mechanism of blockchain regulation

\section{Regulating Blockchain Technology Energy Conservation Standards}

In order to sustain services, blockchain application relies heavily on physical machinery and its cooling equipment. Establishing minimum energy-saving requirements on blockchain-related hardware could be highly successful in increasing its systems' overall efficiency (Truby 2018). It could ease the selection task of distinguishing between efficient and inefficient blockchain machinery for infrastructure designers. Regarding energy conservation standards, data and I.T. centers in Switzerland have started to adopt efficiency standards, which are being fronted by its national data center efficiency association. It awards data and I.T. centers, which achieve excellent efficiency and sustainability scores in its system design and day-to-day operation. These standards also include best practice guidelines based on energy source selection, heat regulation systems, and recycling capabilities. It will provide a guideline for infrastructure designers and operators to adhere to in the future (Judge, 2020). Due to blockchain application's similarities in their energy-intensive 
operation to data centers, it may be beneficial to consider establishing or adopting such energy efficiency requirements for technology operators and designers of this technology (Clark \& Greenley, 2019). It would support the decarbonization targets of the governments and the overall energy efficiency of blockchain technology.

\section{Financial Incentives}

Financial-based incentives on blockchain services dependent on traditional energy sources are another area that can be explored. Implementation of differentiated tax rates based upon the type of energy source relied on by a mining center to enable its operation could spur changes. The increase in cost structure could create an urge for these 'mining centers' to look into renewable to power up their day-to-day operation. The practice is similar to the taxing goods produced using renewable energy lower than a similar product that utilized hydrocarbons. Drawing similarities to data centers, the power consumption of operating and cooling in the industry is tipped to reach a staggering 73 billion kWh in 2020 (Truby 2018 ). For this reason, the data center industry operators are becoming more proactive in minimizing the environmental impact of their facilities. For example, the U.S. Environmental Protection Agency (EPA) introduced the Renewable Energy Production Incentive Program to provide financial incentives such as lower taxes for power generated using solar, wind, geothermal, and biomass resources. Major data center operators such as Equinix, Amazon, Google, and Microsoft have voiced strong commitment to renewable energy investment (Cloudscene, 2018). Implementing such initiatives for blockchain technology may ease the adaptation of energy-efficient resources to power its operations.

\section{Blockchain Energy Efficiency Challenges and Ethical Compliance}

Energy-efficiency improvements for blockchain is essential as it provides the technology with a sustainable future. As mentioned earlier, several areas can be made to ease the mass implementation of the technology. Addressing the challenges of its adaptation is essential, and governments and major industrial players need to play their part in making the technology sustainable. Although the PoS and PoA consensus protocol effectively reduces energy consumption levels and requires far less sophisticated equipment, they tend to create a more controlling and limited environment, which undermines the decentralized nature of the distributed ledger system design (Clark \& Greenley, 2019). For instance, Eutherian has been looking to implement the PoS algorithm since 2014 and has been delaying its move ever since technical problems. The designers of such a consensus algorithm mainly face the following challenges: nothing at stake, long-range attack, and cartel formation within a platform (Mrcy, 2019). PoW is the most used consensus algorithm currently, and the move toward the PoS or PoA consensus mechanism will require more time before being truly ready for the masses.

The rate of renewables penetration and energy sector de-regularization, especially in Malaysia, is still a work in progress. In 2019, the Malaysia Electricity Supply Industry 2.0 (MESI 2.0) was launched; the 10-year plan's primary focus will be on liberalization across the energy industry. It will range from energy sources, grid operation, distribution of electricity as well as creation of an open energy retail market. Besides that, it will also encourage and assist the penetration of renewable energy into the countries energy mix (Gomez, 2018). Talks of utility sector reformation have been ongoing for a long time. The Malaysian government had proposed the introduction of an open and transparent electricity market by 2005 . The plans were halted as certain countries experienced power failure incidents due to their energy sector (Aziz, 2019). The newly launched MESI 2.0 will hopefully be a springboard for RE penetration in Malaysia and provide a sustainable energy source for blockchain technology's growing demand.

Energy efficiency and ethical compliance are two main challenges that drive the industry to adopt blockchain technology. It can record the activity of energy consumption along in the supply chain and the authenticity of the report. The government can gain access to industrial data and reduce the dependency on nonrenewable energy sources.

Although the implementation of tax relives, incentives and technical standards will spur energy efficiency in the technology, the approach and exposure level of individual governments towards blockchain adoption varies widely across the world. For instance, the Chinese government has been intensely interested in blockchain and has adopted technology policies as part of its 5-year plan. Blockchain is now being extensively tested under its administrations, and tax relives and financial benefits are in the pipeline to accelerate technology adaptation (MiGHT, 2019).

The Malaysian government has also recognized that there are many potentials that blockchain represents across different industries and does not intend to fall behind. Although task forces are currently in their infancy stage, they will continue to discuss policies and implement what is needed for the technology (Adilla, 2018). Malaysia needs to leap forward in its efforts to be a key player in the selected areas, with the government's role critical in regulatory and policy aspects of industrial development and opening up market access for firms.

\section{CONCLUSION}

Blockchain, which initially served as the framework for cryptocurrency, is slowly making waves in many industries. The potential of blockchain is not something that can be overlooked, and the technology is well suited to become an enabler of the ongoing fourth industrial revolution. The increasing support and acceptance of governments, industry players, technical experts, and application providers is a positive sign of the technology. The growing energy demand to support blockchain applications is another indicator of its growth and importance globally. Industries are now moving towards sustainability, and blockchain's non-sustainable energy consumption may grow into a large stumbling block for its wide adaptation.

Generally, the discussions on the technology drawback have been centered on energy consumption and have created a low reputation. Some of the areas of improvement mentioned in this paper, such as its consensus protocol, energy 
sources, technical standards, financial incentives, and policies need to be further researched and addressed to provide genuine operational sustainability for the technology. Improvements in these areas will clear up the negative perception regarding its application and provide a good need boost for mass adaptation of the technology in the near future. It is essential to investigate the nexus of blockchain technology adoption, energy efficiency, and ethical compliance on energy reports and sustainability.

\section{ACKNOWLEDGEMENT}

The authors convey their appreciation to the Division of Research \& Innovation, Universiti Malaysia Pahang for funding this study (PDU203220 \& UIC201518).

\section{REFERENCES}

Abdullah, W. S. W., Osman, M., Ab Kadir, M. Z. A., \& Verayiah, R. (2019). The potential and status of renewable energy development in Malaysia. Energies, 12(12), 2437.

Adilla, F. "Government Setup Special Taskforce to Study Blockchain Potential." NST Online, retrived from: www.nst.com.my/business/2018/03/340273/government-setup-special-taskforce-study-blockchain-potential.

Andoni, M., Robu, V., Flynn, D., Abram, S., Geach, D., Jenkins, D., McCallum, P., \& Peacock, A. (2019). Blockchain technology in the energy sector: A systematic review of challenges and opportunities. Renewable and Sustainable Energy Reviews, 100, $143-174$.

Aziz, A. (2019), "Govt to Liberalise Power Industry." The Edge Markets, retreuevd from: www.theedgemarkets.com/article/govtliberalise-power-industry.

Clark, E., C., \& Greenley, L.,H. (2019). Bitcoin, Blockchain, and the Energy Sector, Independently published [August 9, 2019]. Retrieved from https://www.hsdl.org/?view\&did=828211

Balasubramanian, S., Shukla, V., Sethi, J. S., Islam, N., \& Saloum, R. A readiness assessment framework for Blockchain adoption: A healthcare case study. Technological Forecasting and Social Change, 165, 120536.

Baraniuk, C. (2019, July 3). Bitcoin's energy consumption 'equals that of Switzerland'. Retrieved from https://www.bbc.com/news/technology-48853230

Bumblauskas, D., Mann, A., Dugan, B., \& Rittmer, J. (2020). A blockchain use case in food distribution: Do you know where your food has been?. International Journal of Information Management, 52, 102008.

CCN.com (2020), "Spanish Bitcoin Miner Plans 300 MW Solar Plant with Panels from China." Retrived from: www.ccn.com/spanishbitcoin-miner-putting-up-a-300-mw-solar-plant-for-its-mining-operations/.

Cuthbertson, A (2019). "Bitcoin Uses More Energy than the Whole of Switzerland - and It's Getting Worse." The Independent, Independent Digital News and Media, Retrievd from: www.independent.co.uk/life-style/gadgets-and-tech/news/bitcoinenergy-switzerland-cryptocurrency-mining-a8989816.html.

Cloudscene (2018). "The Data Center Sector Is Driving Renewable Energy Adoption." Data Center News, retreievd from: cloudscene.com/news/2018/01/data-center-renewable-energy/.

Eurelectric Report (2018), "Energy Blockchain-Project Featured in EURELECTRIC Report." Center for Secure Energy Informatics, Retrieved from: cdn.eurelectric.org/media/3115/paper1_blockchain_eurelectric-h-CB8D6920.pdf.

Fernando, Y., Rozuar, N. H. M., \& Mergeresa, F. (2021), The blockchain-enabled technology and carbon performance: Insights from early adopters. Technology in Society, 64, 101507.

Fernando, Y., \& Wulansari, P. (2020). Perceived understanding of supply chain integration, communication and teamwork competency in the global manufacturing companies. European Journal of Management and Business Economics. Vol. ahead-of-print No. ahead-of-print.

Fernando, Y., Abideen, A. Z., \& Shaharudin, M. S. (2020a). The nexus of information sharing, technology capability and inventory efficiency. Journal of Global Operations and Strategic Sourcing, 33(4), 327-351.

Fernando, Y., Darun, M. R., Al-haimi, B., Ibrahim, D. N., Tieman, M., \& Mohamad, F. (2020b), Role of Smart Contracts in Halal Supply Chain Management. In Encyclopedia of Organizational Knowledge, Administration, and Technology, 2497-2504.

Fernando, Y., Darun, M. R., Abideen, A. Z., Ibrahim, D. N., Tieman, M., \& Mohamad, F. (2020c), Adoption of Blockchain Technology to Improve Integrity of Halal Supply Chain Management. In Encyclopedia of Organizational Knowledge, Administration, and Technology, 2488-2496.

Gielen, D., Boshell, F., Saygin, D., Bazilian, M. D., Wagner, N., \& Gorini, R. (2019). The role of renewable energy in the global energy transformation. Energy Strategy Reviews, 24, 38-50.

Gomez, O., C. (20198), "'Deregulate Renewables, Power Generation'." The Edge Markets, retrievd from: www.theedgemarkets.com/article/deregulate-renewables-power-generation.

Irena (2016), Letting in the Light: How solar photovoltaics will revolutionise the electricity system. (2016). Retrieved from https://www.irena.org/publications/2016/Jun/Letting-in-the-Light-How-solar-photovoltaics-will-revolutionise-theelectricity-system

IEEE (2019), IEEE Spectrum: Technology, Engineering, and Science News, retrived from: spectrum.iee.org/computing/networks/ethereum-plans-to-cut-its-absurd-energy-consumption-by-99-percent.

Judge, P. (2020), "Swiss Data Center Efficiency Label Launched at WEF." Retrieved from: www.datacenterdynamics.com/en/news/swiss-data-center-efficiency-label-launched-wef/.

Khatoon, A., Verma, P., Southernwood, J., Massey, B., \& Corcoran, P. (2019). Blockchain in Energy Efficiency: Potential Applications and Benefits. Energies, 12(17), 3317. doi: 10.3390/en12173317

Lohmer, J., \& Lasch, R. (2020). Blockchain in operations management and manufacturing: Potential and barriers. Computers \& Industrial Engineering, 149, 106789.

Mohter, N., \& Fernando, Y. (2020). Corporate Governance and Low Carbon Supply Chains: Why Integrity Matters?. Journal of Governance and Integrity, 3(2), 39-45.

Mrcy, P.L. (2019). "Why Isn't Ethereum on PoS Already?" Medium, IBBC.io, 8 Jan. 2019, retrievd from: medium.com/ibbc-io/the- 
beautiful-complexity-of-pos-338cfc340eaa.

MiGHT (2019), "Malaysia Blockchain \& Distributed Ledger (DLT) Outlook 2019." MIGHT Portal, retrievd from: www.might.org.my/malaysia-blockchain-distributed-ledger-dlt-outlook-2019/.

Monrat, A. A., Schelén, O., \& Andersson, K. (2019). A survey of Blockchain from the perspectives of applications, challenges, and opportunities. IEEE Access, 7, 117134-117151.

Naina, L. M., Fernando, Y., \& Shaharudin, M. S. (2019). Extended Eco-Efficient Supply Chain Integration Management Model: A Research Note. Journal of Governance and Integrity, 3(1), 69-76.

PricewaterhouseCoopers. (2016). Blockchain-an opportunity for energy producers and consumers? Retrieved from https://www.pwc.com/gx/en/industries/energy-utilities-resources/publications/opportunity-for-energy-producers.html

Sharvini, S. R., Noor, Z. Z., Chong, C. S., Stringer, L. C., \& Yusuf, R. O. (2018). Energy consumption trends and their linkages with renewable energy policies in East and Southeast Asian countries: Challenges and opportunities. Sustainable Environment Research, 28(6), 257-266.

SEDA (2020), Renewable Energy Malaysia. Retrieved from http://www.seda.gov.my/reportal/

Tan, C. S., Maragatham, K., \& Leong, Y. P. (2013). Electricity energy outlook in Malaysia. In IOP Conference Series: Earth and Environmental Science, 16(1), p. 012126.

Truby, J. (2018). Decarbonizing Bitcoin: Law and policy choices for reducing the energy consumption of Blockchain technologies and digital currencies. Energy research \& social science, 44, 399-410.

Shaharudin, M. S., Fernando, Y., Ahmed, E. R., \& Shahudin, F. (2020). Environmental NGOs Involvement in Dismantling Illegal Plastic Recycling Factory Operations in Malaysia. Journal of Governance and Integrity, 4(1), 29-36.

Wu, J., \& Tran, N. (2018). Application of Blockchain Technology in Sustainable Energy Systems: An Overview. Sustainability, 10(9), 3067.

Yaga, D., Mell, P., Roby, N., \& Scarfone, K. (2018). NISTIR 8202 Blockchain Technology Overview. National Institute of Standards and Technology. Retrieved from: https://nvlpubs. nist. gov/nistpubs/ir/2018/NIST. I.R., 8202.

Zhang, S., \& Lee, J. H. (2020). Analysis of the main consensus protocols of Blockchain. ICT express, 6(2), 93-97.

Zheng, Y., \& Boh, W.F. (2021), Value Drivers of Blockchain Technology: A Case Study of Blockchain-Enabled Online Community, Telematics and Informatics, 58, 101563.

\section{Conflict of Interest}

There is no conflict of interest.

\section{Authors' Biography}

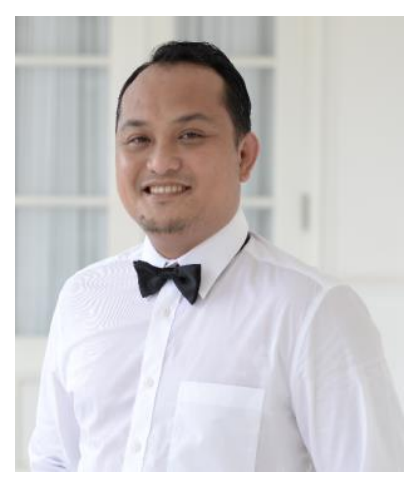

Yudi Fernando holds a Ph.D. and Associate Professor. He is the former Deputy Dean of Research and Postgraduate Studies at the Faculty of Industrial Management, Universiti Malaysia Pahang. He has experience working in the electronics industry for several years. His current research interests are sustainable supply chain management, halal logistics, blockchain technology, and service management. He is also a member of the Society of Logisticians, Malaysia/Pertubuhan Pakar Logistik Malaysia (LogM).

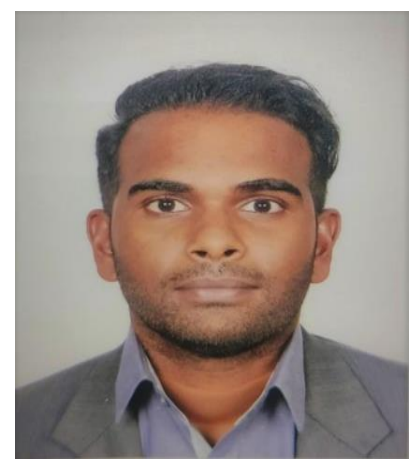

Ir. Rubakanthan Saravannan is an Electrical Engineer at Tenaga Nasional Berhad. He is a Professional Engineer (P.Eng) registered with the Board of Engineers Malaysia and certified 132kV Competent Electrical Engineer by Energy Commission of Malaysia. His experience and interest are predominantly in operation and maintenance of grid connected high voltage electrical substation. Email: rubakanthan@tnb.com.my 